Supporting information to

\title{
Physical Strategy to Determine Absolute Electrochemiluminescence Quantum Efficiencies of Coreactant Systems Using a Photon-counting Photomultiplier Device
}

Kenneth Chu, Jonathan R. Adsetts, Jing Ma, Congyang Zhang, Mahdi Hesari, Liuqing Yang, Zhifeng Ding *

Department of Chemistry, Western University, 1151 Richmond Street, London, ON, N6A 5B7, Canada.

*Corresponding author: zfding@uwo.ca 


\section{Table of Contents}

Sample calculation for the absolute quantum efficiency. .................................................... S-2

Photographs of ECL cell with its holder and photon counting head assembly....................S-4

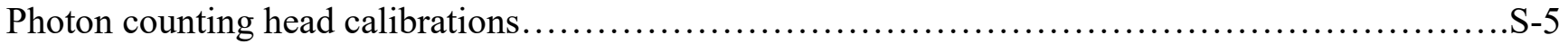

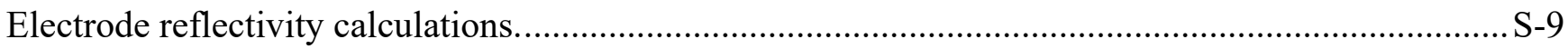

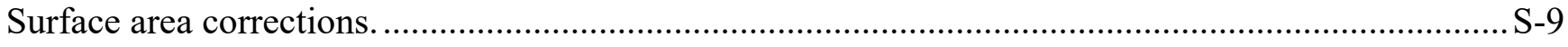

Principles of photomultiplier tube operation in photon-counting mode........................................ S-10

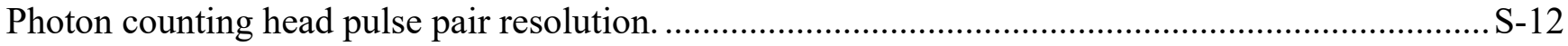

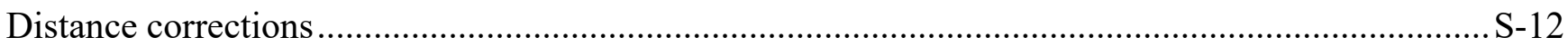

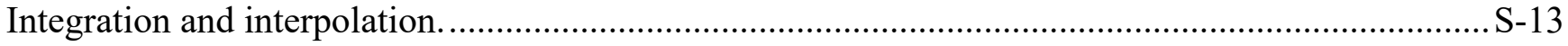

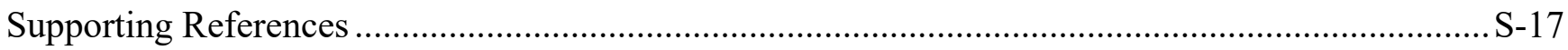

Sample calculation for the absolute quantum efficiency. Equations $\mathrm{S} 1$ to $\mathrm{S} 9$ demonstrate a full calculation of the absolute EQE for $\mathrm{Ru}(\mathrm{bpy}) 3^{2+}$ annihilation ECL during a potential sweep (as in Figure 4 in the main text). Equation S1: integration of the counts with respect to the bin number, multiplied by the bin width, results in total counts. Equation S2: dividing by the $\mathrm{C}$ value results in total photons measured by the PCH. Equation S3: calibration factor corrects to a calibrated number of photons. Equation S4: surface area correction factor. Equation S5: Electrode reflectance correction factor. Equation S6: solution self-absorbance correction factor. Equation S7: integration of the measured current with respect to time results in the total charge. Equation S8: dividing the total charge by the elementary charge constant $\left(e=1.602 \times 10^{-19} \mathrm{C}\right)$ yields total number of electrons. Equation S9: the ratio between the total photons vs. the total electrons results in the absolute quantum efficiency, expressed here as a percentage. Unless otherwise specified, integration limits are assumed to be from 0 $=$ start of potentiodynamic experiment to $t=$ end of potentiodynamic experiment, inclusive. 


$$
\begin{aligned}
& \text { Total counts }=\int_{0}^{t} \text { Counts } \cdot d t=483000 \text { counts } \\
& 483000 \text { counts } \times \frac{1}{0.0564 \frac{\text { counts }}{\text { photon }}}=8.56 \times 10^{6} \text { photons } \\
& 8.56 \times 10^{6} \text { photons } \times \frac{1}{0.0769}=1.11 \times 10^{8} \text { photons } \\
& 1.11 \times 10^{8} \text { photons } \times \frac{1}{9.65 \times 10^{-4}}=1.15 \times 10^{11} \text { photons } \\
& \frac{1.15 \times 10^{11} \text { photons }}{1.40}=8.24 \times 10^{10} \text { photons } \\
& 8.24 \times 10^{10} \text { photons } \times 1.1952=9.85 \times 10^{10} \text { photons } \\
& \text { Total charge }=\int_{0}^{t} \text { Current } \cdot d t=4.38 \times 10^{-4} \text { Coulombs } \\
& \text { Total electrons }=\frac{\text { Total charge }}{e}=\frac{4.38 \times 10^{-4} C}{1.602 \times 10^{-19} \frac{C}{\text { electron }}}=2.73 \times 10^{15} \text { electrons } \\
& E Q E \%=\frac{\text { Total photons }}{\text { Total electrons }} \times 100=\frac{9.85 \times 10^{10} \text { photons }}{2.73 \times 10^{15} \text { electrons }} \times 100=\mathbf{0 . 0 0 3 6} \%
\end{aligned}
$$


Photographs of ECL cell with its holder and photon counting head assembly.

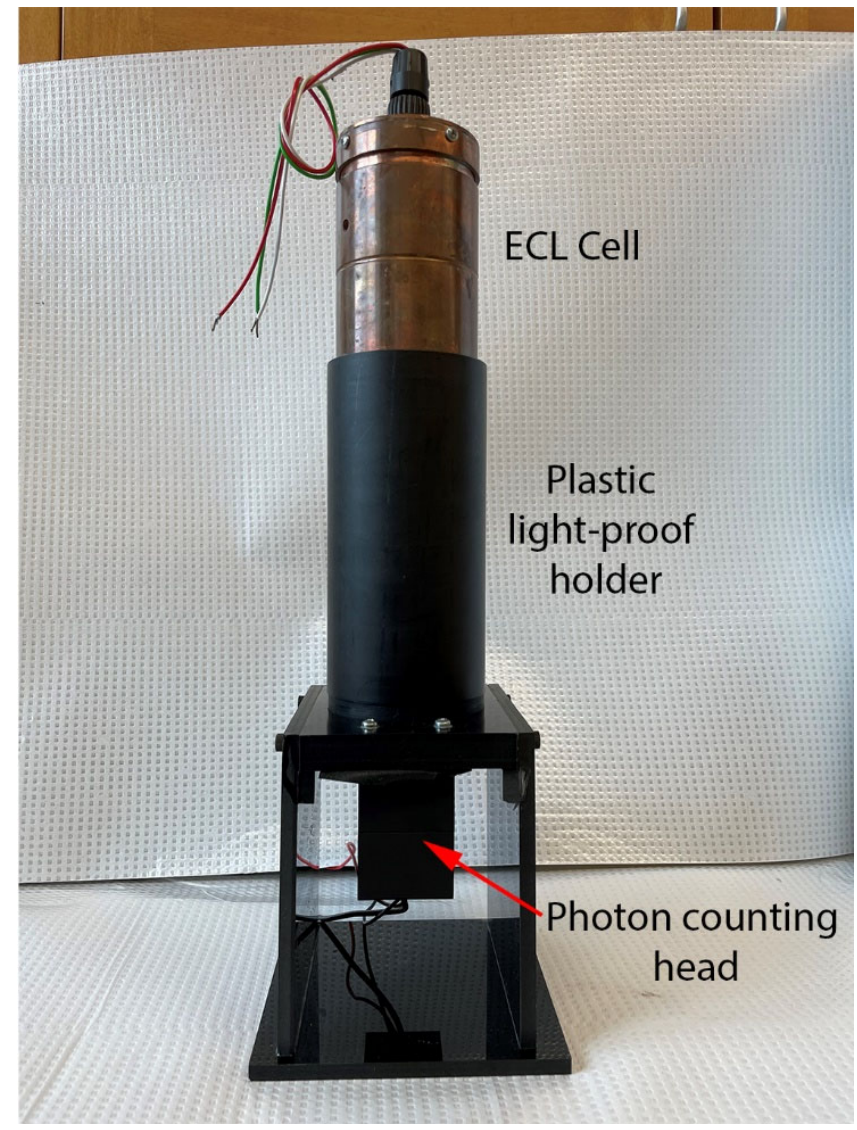

Figure S1. Photograph of the ECL cell in its black plastic light-proof holder, and the photon counting head. 


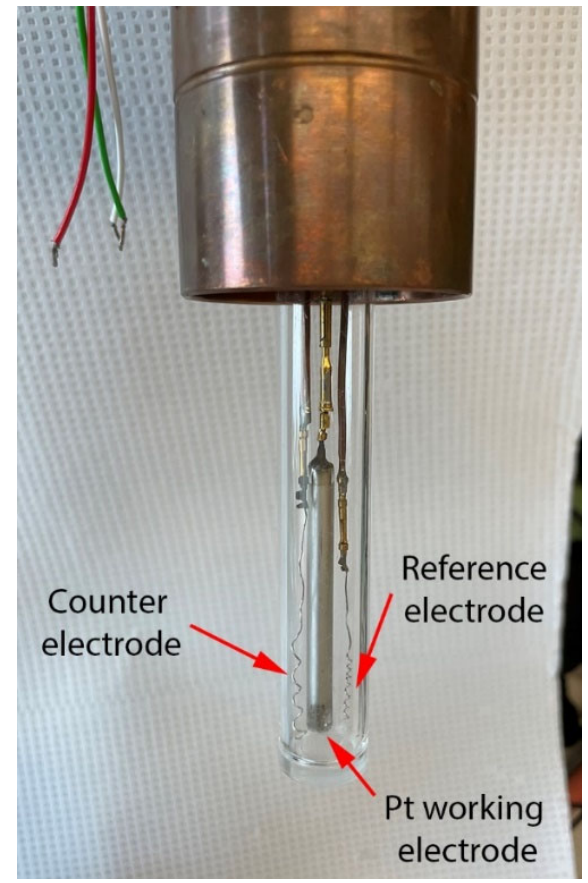

Figure S2. Photograph of the ECL glass cell and its assembly, showing the orientation of the working, counter, and reference electrodes.

Photon counting head calibrations.

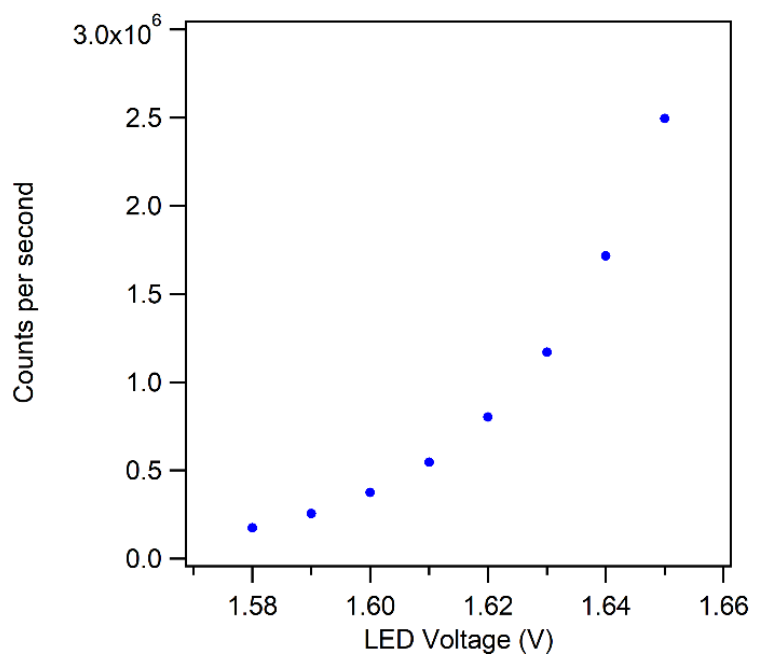

Figure S3. Counts per second (corrected for bin widths) of a red LED measured using the Hamamatsu H6420-02 photon counting head at different operating voltages $(1.58 \mathrm{~V}-1.65 \mathrm{~V})$. 


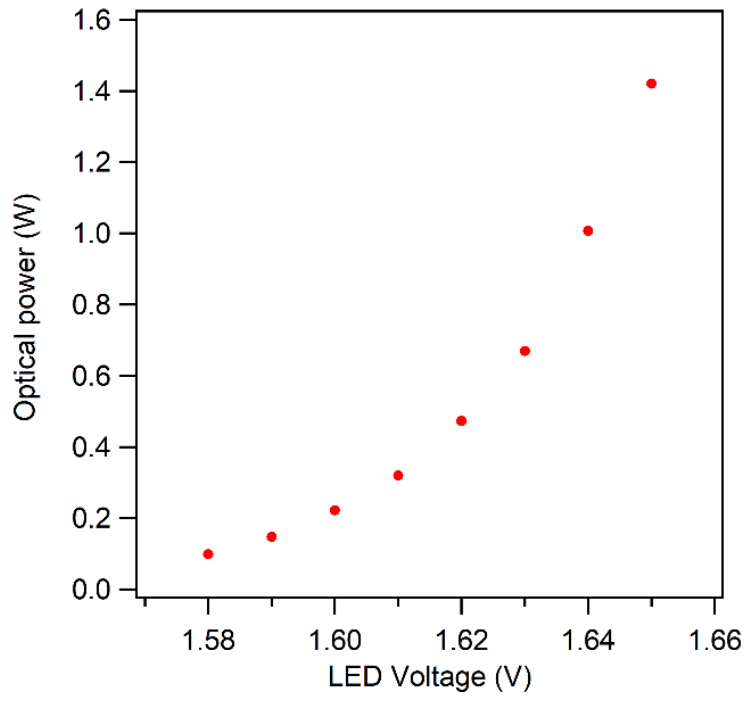

Figure S4. Optical power (in Watts) of a red LED measured using a Thorlabs calibrated silicon photodiode at different operating voltages $(1.58 \mathrm{~V}-1.65 \mathrm{~V})$.

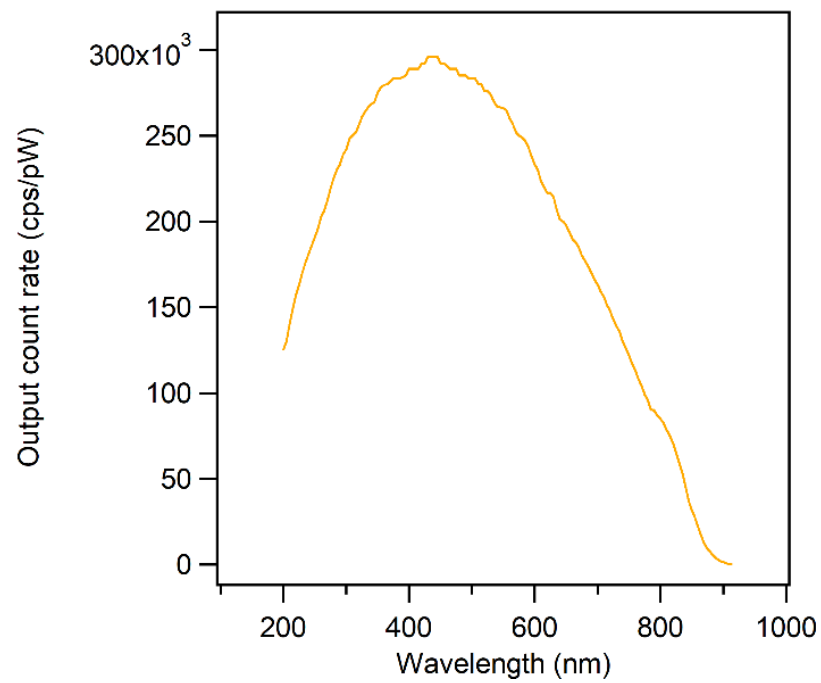

Figure S5. Quantum efficiency of the Hamamatsu H6420-02 photon counting head (measured in $\mathrm{cps} / \mathrm{pW}$ ) vs. the incoming emission wavelength. 
The quantity cps/pW is equivalent to counts/incident photon, if the energy of the photons are considered. The following equations were used to relate a photon's energy with its wavelength, where $h$ is the Planck constant $\left(1.986 \times 10^{-25} \mathrm{~J} \cdot \mathrm{m}\right), c$ is the speed of light $\left(3.00 \times 10^{8} \mathrm{msec}^{-1}\right)$ and $\lambda$ is the wavelength in meters. In this way, subsequent calculations can be performed with more consistent units.

$$
\begin{gathered}
E=\frac{h c}{\lambda}=J \text { per photon }(\lambda) \\
W=\frac{J}{S} \times \frac{1}{\frac{J}{\text { photon }(\lambda)}}=\frac{\text { photon }(\lambda)}{s} \\
\frac{\frac{\text { counts }}{s}}{p W}=\frac{\frac{\text { counts }}{S}}{\frac{\text { photons }}{S}}=\frac{\text { counts }}{\text { photon }}
\end{gathered}
$$

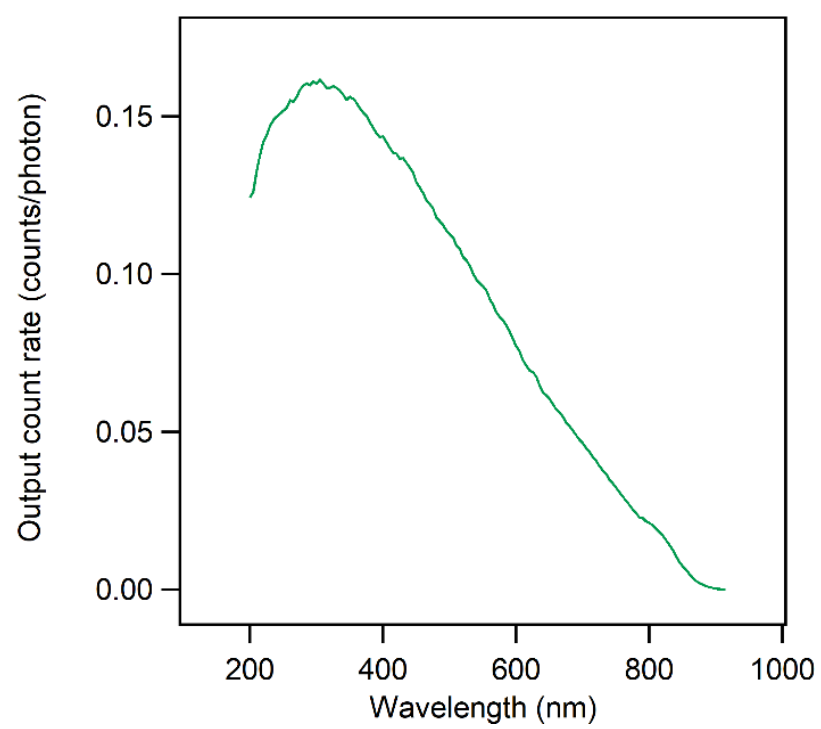

Figure S6. Quantum efficiency of the Hamamatsu H6420-02 photon counting head (measured in counts/incident photon) vs. the incoming emission wavelength. 


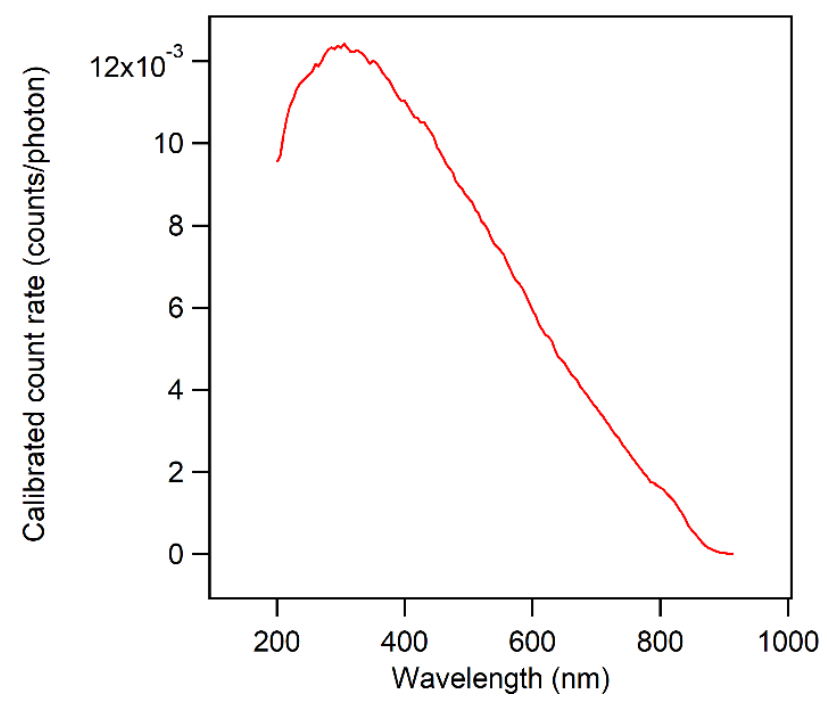

Figure S7. Calibrated quantum efficiency of the Hamamatsu H6420-02 photon counting head (measured in counts/incident photon) vs. the incoming emission wavelength. Obtained by applying the calibration factor to Figure S6.

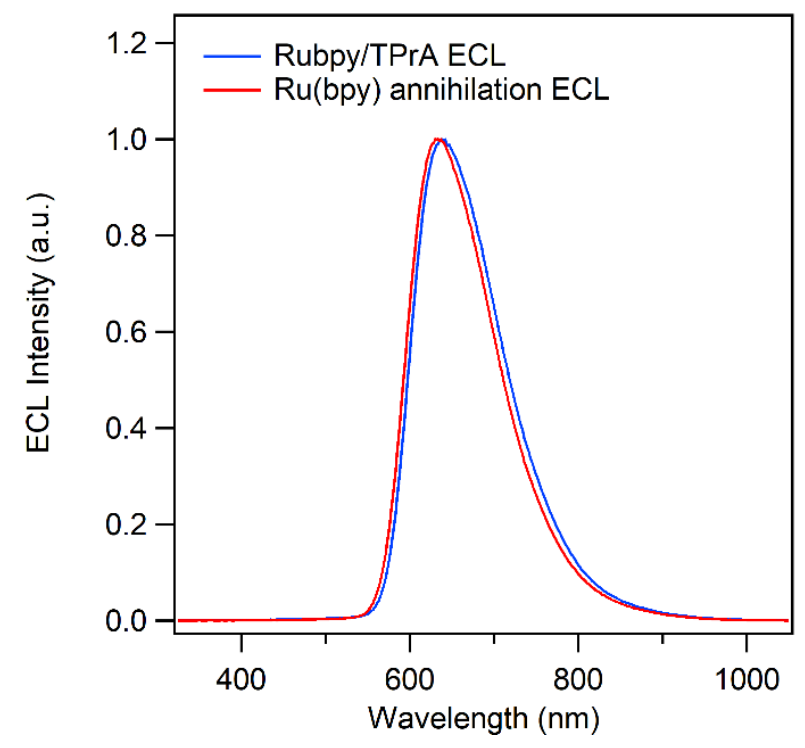

Figure S8. Background-corrected and normalized intensity ECL emission spectra of $\mathrm{Ru}(\mathrm{bpy}) 3^{2+}$ annihilation and TPrA coreactant systems. 
Electrode reflectivity calculations. We first consider the two bounding scenarios of electrode reflectivity. The first occurs if the electrode surface absorbs all incident radiation; here, a correction factor of 2 is required to account for the lost photons projected towards the upper hemisphere. The second scenario occurs if the electrode surface is instead a perfect mirror, in which case no correction factor is required, because all the ECL photons will eventually end up at the photodetector. The experimental correction factor will therefore lie somewhere in between these two extremes. For the experiments described in this work, the following equation (Equation S13) provides the reflectivity correction factor $(\sigma)$, where ECL corrected and ECLobserved is the corrected and observed ECL intensity, respectively, and $R$ is the reflectivity of the electrode (assumed to be $60 \%$ ).

$$
\sigma=\frac{E C L_{\text {corrected }}}{E C L_{\text {observed }}}=2-R=2-0.6=1.4
$$

\section{Surface area corrections.}
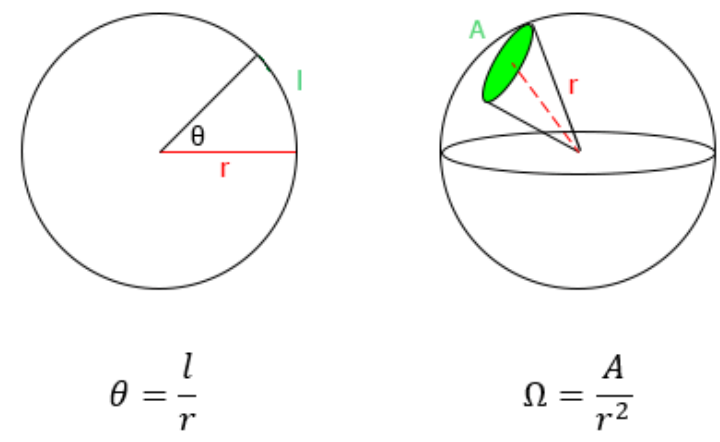

Figure S9. A comparison of angles (circles) vs. solid angles (spheres). A circle contains $2 \pi$ radians, while a sphere contains $4 \pi$ steradians. The solid angle (in steradians) of a unit sphere is analogous to the planar angle (in radians) of a unit circle. Radians = ratio of the length of the arc vs. radius of the circle. Steradians $=$ ratio of the spherical surface area vs. the square of the radius of the sphere. ${ }^{1}$ 
Principles of photomultiplier tube operation in photon-counting mode. A photomultiplier tube (commonly, PMT) produces photoelectrons as a response to incoming photons incident at the photocathode. These photoelectrons are accelerated towards a series of charged metal electrodes (dynodes), where they are multiplied via secondary electron emission. This process is repeated several times, depending on the number of dynodes present, resulting in an overall gain factor usually on the order of one million for typical photomultipliers. These amplified photoelectrons are finally collected at the anode as a current pulse (Figure S10).

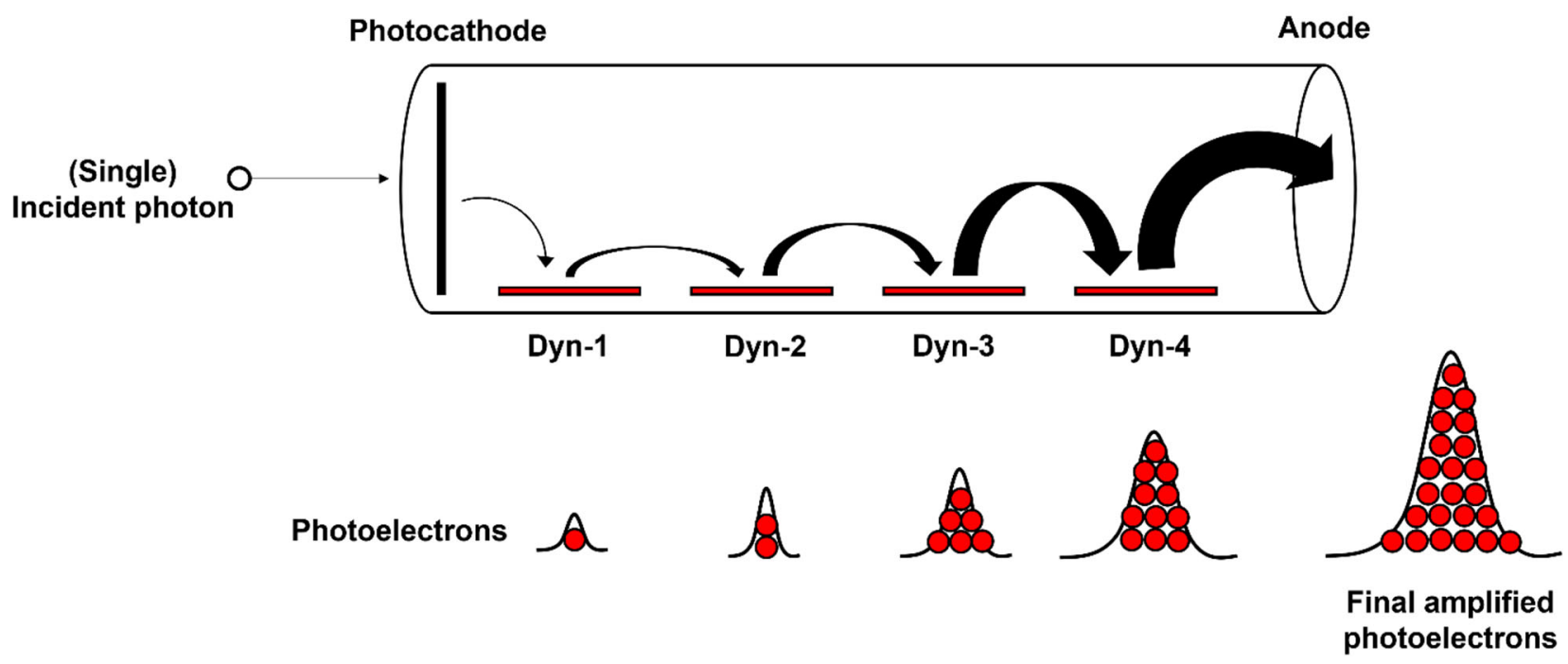

Figure S10. Operating principles of photomultipliers. Figure is for illustrating the concept only: number of dynodes and amplification level does not represent an actual photomultiplier.

In general, there are two operational modes for PMTs. If the number of incident photons is relatively high (where the pulse-pair resolution is insufficient to resolve individual current pulses, or if the signal processing circuits are otherwise too slow to respond), then overlap of the output current can occur. This results in a continuous direct current (often referred to as the output photocurrent). This mode is sometimes called multi photon counting (Figure S11). 
(a)

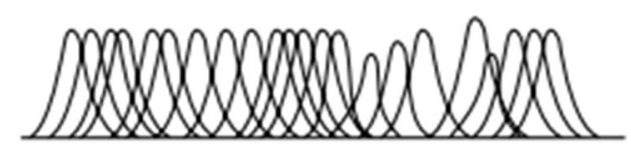

TIME

(b)

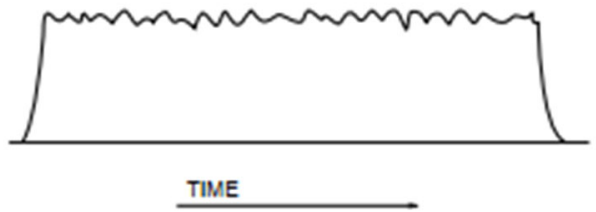

Figure S11. Multi-photon counting mode: a) amplified photoelectrons, and b) current output at the photomultiplier anode.

However, if the incoming light intensity is low, the incident photons may appear as discrete pulses of current from the anode (Figure S12). This mode is sometimes called single photon counting. This technique allows for very high sensitivity and superior signal to noise, because the number of pulses is directly proportional to the number of incident photons.
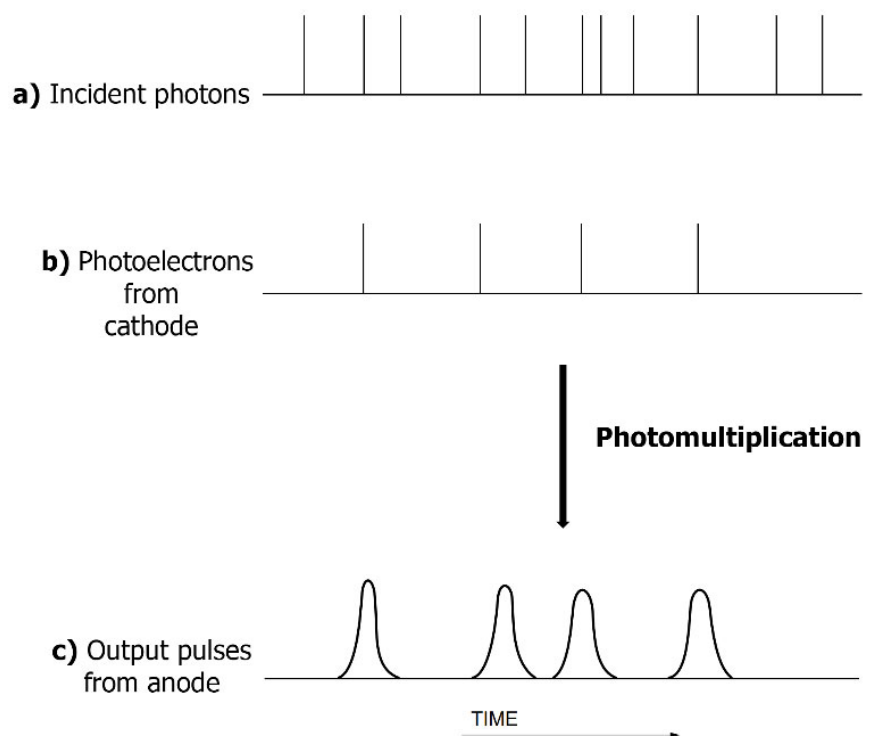

Figure S12. Single photon counting mode. Incident photons strike the photocathode (a), and photoelectrons are emitted (b). Cascading multiplication of photoelectrons through the dynodes results in an amplified signal that arrives at the anode (c). 
A key challenge with single photon-counting is background noise elimination. This is addressed with the incorporation of a discriminator module that can distinguish between photon pulses that are due to background noise and photon pulses that originate from the system of interest. This can be typically accomplished by comparing the output pulse height; since background photons tend to have lower overall multiplication factors, setting an appropriate discriminator level will eliminate most 'dark noise'. The pulses that successfully pass the discriminator are then sent to a pulse shaper, which adjusts the output pulses to have a constant pulse width. Finally, these pulses can be routed into an instrument capable of high frequency counting (in this work, the SR430 instrument). Figures S11 and S12 were adapted from the Hamamatsu Photonics product catalog. ${ }^{2}$

Photon counting head pulse pair resolution. The pulse-pair resolution of the photon counting head describes the minimum time interval over which two pulses will appear as discrete signals, i.e. there will be no overlap between subsequent pulses. The true counting rate $(N)$ can be estimated using the following equation, where $M$ is the measured counting rate and $t$ is the time resolution.

$$
N=\frac{M}{1-M t}
$$

Distance corrections. To compensate for emission losses due to their distances from the photodetector, the following equations were used, where $R$ is the radius of the photodetector (in general equal to $\sqrt{ }(\mathrm{A} / \pi))$ and $D$ is the distance between the photodetector and the emission source. The resulting solid angle $(\Omega)$ has units of steradians.

$$
\Omega=2 \pi(1-\cos \theta)=2 \pi\left(1-\cos \frac{R}{D}\right)
$$

In all measurements described in this work, we have, wherever possible, adhered to the 'five times rule', which allows the approximation of an emitter as a point source if the distance to the detector is greater than five times the largest dimension of the light source. ${ }^{3}$ For example: the largest dimension on the platinum electrode (emitter source) is $2 \mathrm{~mm}$, so the detector should be placed at a distance $>10 \mathrm{~mm}$. 
Integration and interpolation. All integration procedures described in this work were performed with the assistance of MATLAB programming software (version 2018b, MathWorks Inc., Natick, MA) and the cumulative trapezoidal numerical integration (cumtrapz) approximative function with unit spacing. Integration steps required an additional correction factor equal to the bin width for PCH measurements, and data interval for Autolab electrochemical measurements in order to perform the integration with respect to time.

$$
\begin{gathered}
\text { Total photons }=\frac{d t}{d x}[\text { Bin Width }] \times \int_{0}^{x} \text { Photon flux } \cdot d x \\
\text { Total charge }=\frac{d t}{d y}[\text { Data interval }] \times \int_{0}^{y} \text { Current } \cdot d y
\end{gathered}
$$

Interpolation was carried out using the one-dimensional interpolation function in MATLAB (interp1) with the default linear method, for scenarios where two separate sets of data required identical intervals and limits, i.e.. the calculation of the $C$ value. The linear method produces an interpolated value between at least two input values using a straight line approximation of the data. Using this function, we generate a new interpolated data array for both the ECL emission spectrum of interest and the photodetector responsivity curve with precisely defined intervals of $\mathrm{d} \lambda$. This allows the subsequent integration procedures to be performed with respect to this defined $\mathrm{d} \lambda$. Figure S13 shows an excellent agreement between the measured ECL emission spectrum and the interpolated spectrum. 


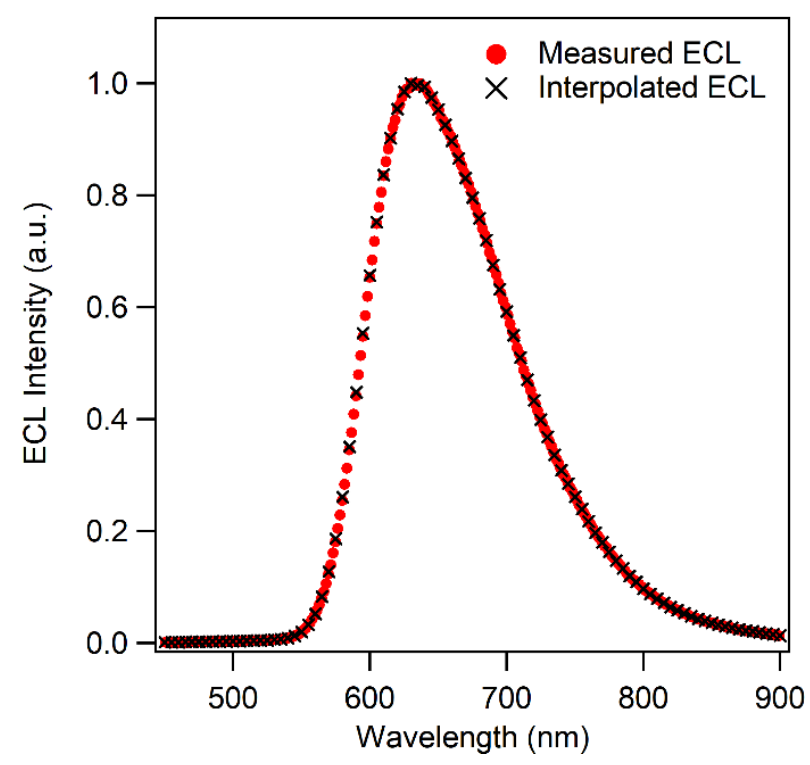

Figure S13. Comparison between measured ECL emission spectrum and resampled ECL emission spectrum generated using the interpl function (default linear option) in MATLAB.

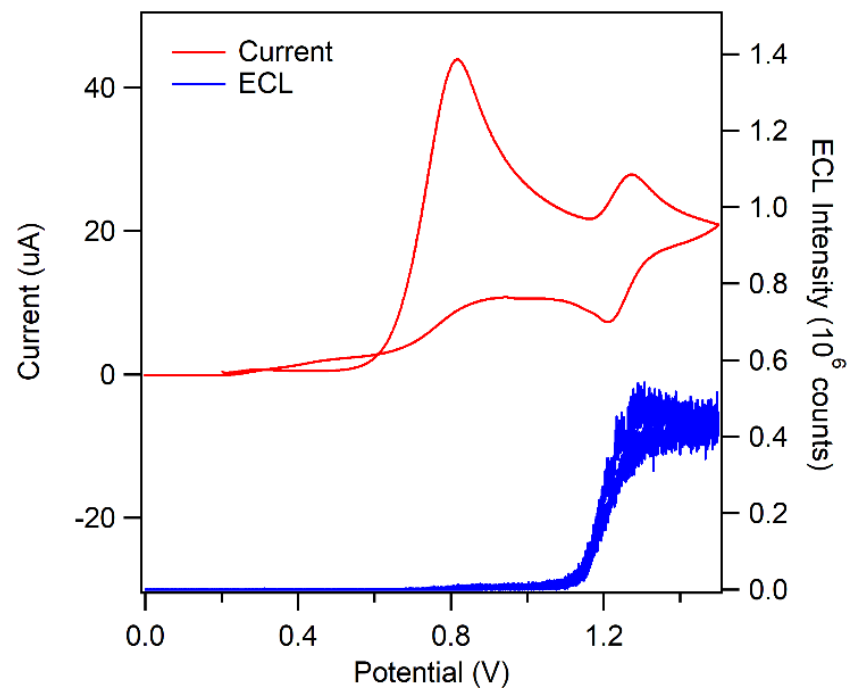

Figure S14. Cyclic voltammogram (red) and ECL voltage curve (blue) of $1 \mathrm{mM}$ Ru(bpy) in acetonitrile with $5 \mathrm{mM}$ TPrA. Scan rate for the cyclic voltammogram was $100 \mathrm{mV} / \mathrm{sec}$. Bin width $=2.62 \mathrm{msec}$, number of bins $=11264$, trigger level $=0.30 \mathrm{~V}$, records per scan $=1$. 


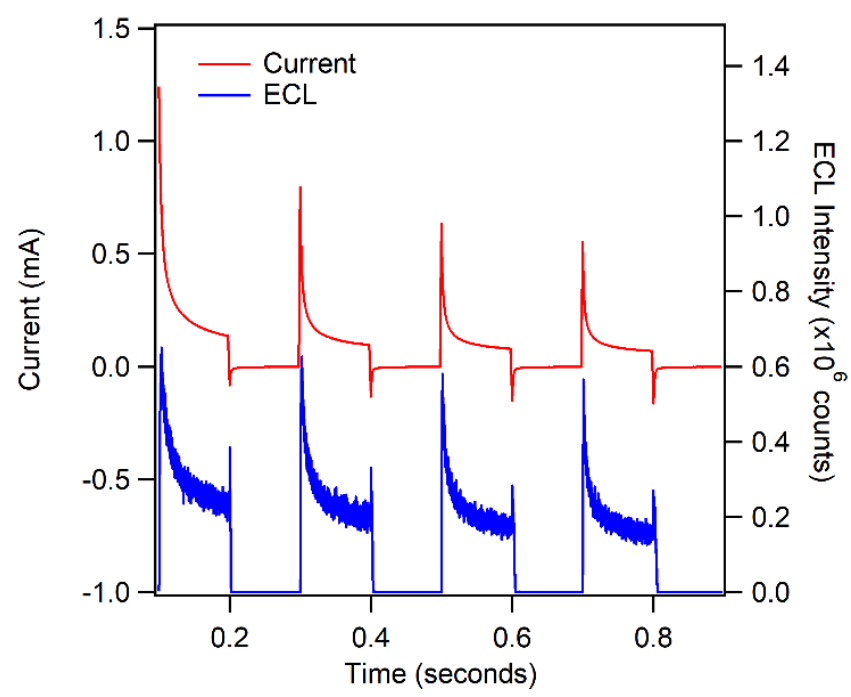

Figure S15. $\mathrm{Ru}(\mathrm{bpy}) 3^{2+}$ pulsing $10 \mathrm{~Hz}, 5 \mathrm{mM}$ TPrA. Bin width $=81.92 \mu \mathrm{sec}$, number of bins $=13312$, trigger level $=-1.40 \mathrm{~V}$, records per scan $=1$.

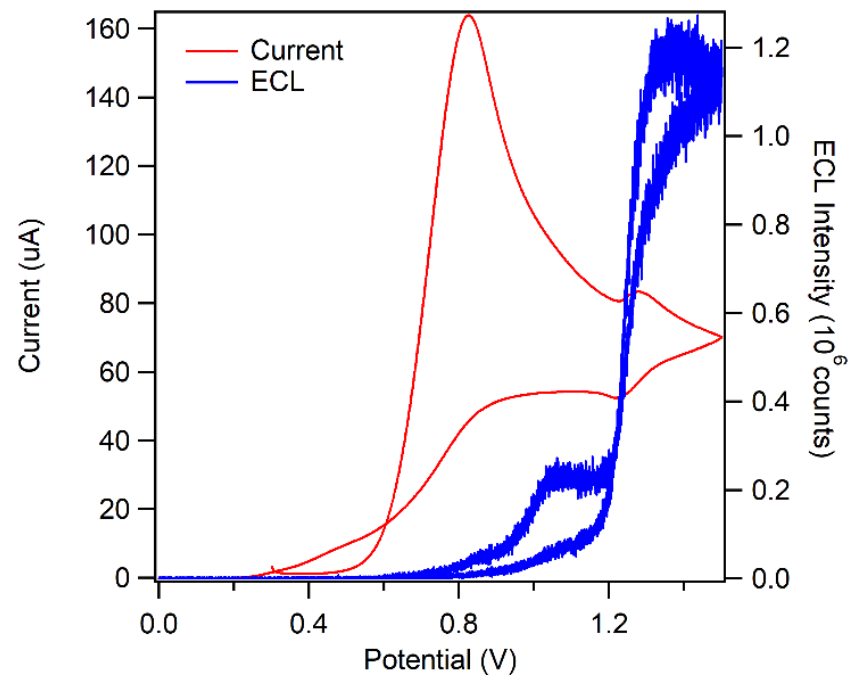

Figure S16. $\mathrm{Ru}(\mathrm{bpy}) 3^{2+} \mathrm{CV}, 20 \mathrm{mM}$ TPrA. Bin width $=2.62 \mathrm{msec}$, number of bins $=11264$, trigger level $=0.30 \mathrm{~V}$, records per scan $=1$. 


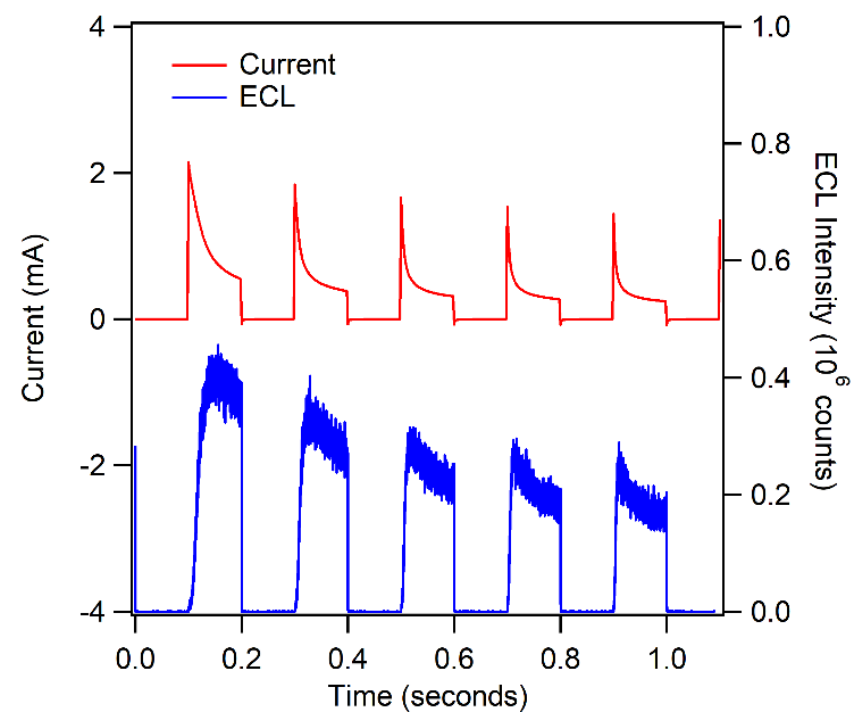

Figure S17. $\mathrm{Ru}(\mathrm{bpy}) 3^{2+}$ pulsing $10 \mathrm{~Hz}, 20 \mathrm{mM}$ TPrA. Bin width $=81.92 \mu \mathrm{sec}$, number of bins $=13$ 312 , trigger level $=-1.40 \mathrm{~V}$, records per scan $=1$.

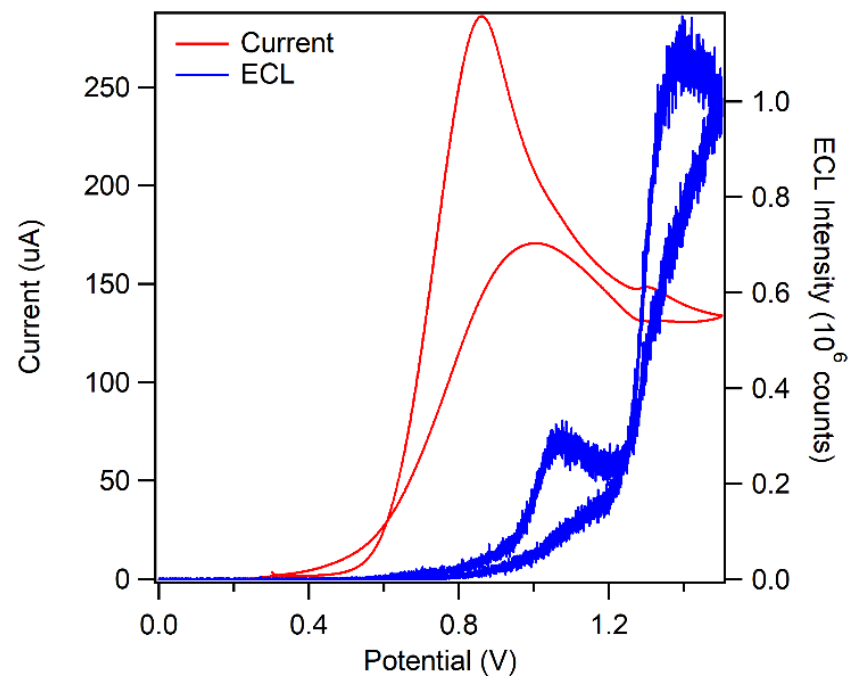

Figure S18. $\mathrm{Ru}(\mathrm{bpy}) 3^{2+} \mathrm{CV}, 50 \mathrm{mM}$ TPrA. Bin width $=2.62 \mathrm{msec}$, number of bins $=11264$, trigger level $=0.30 \mathrm{~V}$, records per scan $=1$. 


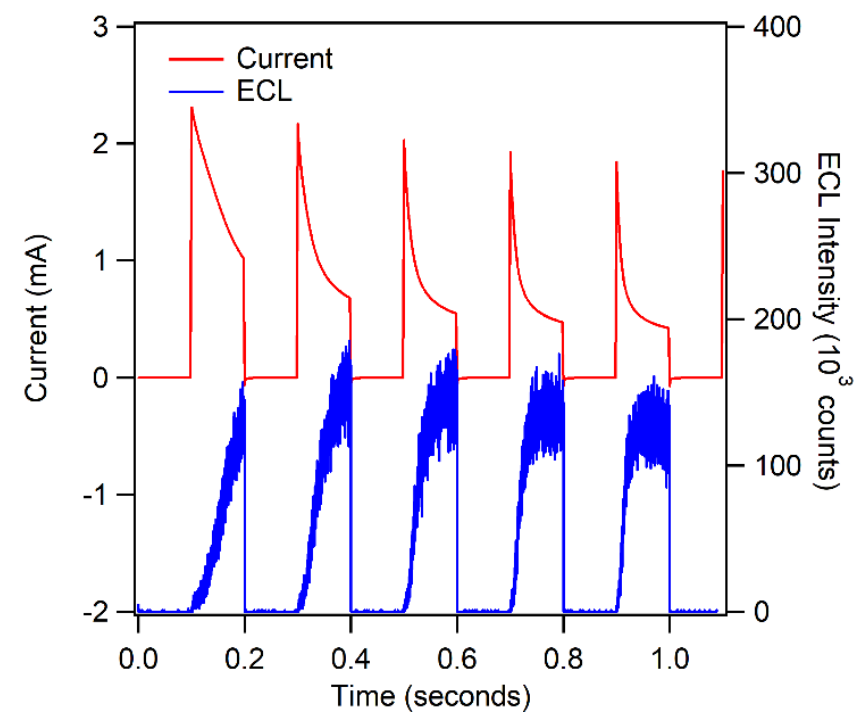

Figure S19. $\mathrm{Ru}(\mathrm{bpy}) 3^{2+}$ pulsing $10 \mathrm{~Hz}, 50 \mathrm{mM}$ TPrA. Bin width $=81.92 \mu \mathrm{sec}$, number of bins $=13$ 312 , trigger level $=-1.40 \mathrm{~V}$, records per scan $=1$.

\section{Supporting References:}

1. Eriksson, F., On the Measure of Solid Angles. Mathematics Magazine 1990, 63, 184-187.

2. Hakamata, T. et al., Photomultiplier Tubes. $3^{\text {rd }}$ ed., Hamamatsu, 2007.

3. Ryer, A. D., Light Measurement Handbook; International Light: Newburyport, MA, 1997. 